\title{
Diseño, Construcción y Evaluación de una Sembradora de Semilla Desnuda para el Cultivo de Tabaco.
}

\section{Design, Construction and Evaluation of a Naked Seed Planter for the Cultivation of Tobacco.}

\author{
Julián Cesar Velásquez R. ${ }^{1}$ y Jhon Fredy Rivera T. ${ }^{2}$
}

\begin{abstract}
Resumen
En la presente investigación se exponen las etapas de diseño y construcción de una sembradora de semilla desnuda y su evaluación frente a la siembra tradicional por regadera utilizada en las zonas tabacaleras del departamento del Huila. Como parámetros fundamentales, se determinó el diámetro promedio de la semilla de tabaco mediante juego de tamices de granulometría de suelos para conocer el tamaño de salida de las boquillas y con el cálculo de los ángulos de deslizamiento y reposo se estableció el talud de la tolva. Para la evaluación se contó con 40 bandejas plásticas de 128 alvéolos, 20 para cada implemento sumando un área total de $6.6 \mathrm{~m} 2$, se tomaron registros del tiempo total de la labor, las semillas depositadas por alveolo, los alveolos sin semilla, el porcentaje de germinación y plántulas a ralear. La sembradora manual produjo un ahorro del $44 \%$ en el costo total de la labor y un 50,4\% en el tiempo empleado para la siembra de semilla en bandejas, además de una reducción del 79,3\% en la cantidad de plántulas a ralear.
\end{abstract}

Palabras Clave: Semilla; Diámetro Promedio; Porcentaje de Germinación; Plántulas a Ralear.

\begin{abstract}
In this research presents the design and construction stages of a naked seed planter and assessing them against traditional planting shower used in the tobacco fields of the department of Huila. As basic parameters, it was determined the average diameter of the seed of snuff through sieve set of soil particle size to determine the output size of the jets and to calculate the slip angles and repose established the slope of the hopper. For the evaluation had 40 plastic trays of 128 alveoli, adding 20 for each implement a total area of $6.6 \mathrm{~m} 2$, were taken records of the total time of work, the seeds deposited in alveoli, the alveoli without seed, the germination percentage and thinning seedlings. The drill manual was a $44 \%$ savings in total cost of the work and $50.4 \%$ in the time used for sowing seeds in trays, plus a $79.3 \%$ reduction in the number of seedlings to thin.
\end{abstract}

Keywords: Seed, Average Diameter, Percentage of Germination, Seedlings to Thin.

\footnotetext{
1 Ingeniero Agrónomo. Esp. Mecanización Agropecuaria. Docente Universidad Surcolombiana Neiva. Avenida Pastrana Carrera $1^{a}$.juvela@usco.edu.co

2 Ingeniero Agrícola. Universidad Surcolombiana Neiva. Avenida Pastrana Carrera 1ª.frejhori@hotmail.com

3
}

Revista Ingeniería y Región No. 7 Año 2010 


\section{Introducción}

Para la siembra de semilla en bandejas es recomendable utilizar semilla pelletizada debido a que brinda un diámetro uniforme para la calibración de boquillas en caso de usar sembradoras mecánicas o neumáticas, además este tipo de semillas proporcionan porcentajes de germinación superiores al $90 \%$. Para la siembra de semilla desnuda se debe optar por métodos que realicen mezclas con otros componentes con el ánimo de aumentar la uniformidad de distribución en las bandejas ya que la idea es usar eficientemente nuestra semilla certificada aplicando una o dos por alveolo.

Inta (2003) menciona que en caso de no tener ninguna posibilidad de acceder a semilla pelletizada es posible sembrar las bandejas con semilla sin pelletizar, tratando de ajustar al máximo la cantidad de semillas en la celda. Una vez que las plantitas llegan a un tamaño razonable (15 a 30 días dependiendo de la temperatura) es necesario ralear las plantitas dejando sólo una por celda y/o trasplantar las pequeñas plantitas a nuevas bandejas (una por celda) hasta completar el ciclo.

Convencionalmente en las zonas tabacaleras del Huila se siembra mediante la "riega de semilla", método en el que se utiliza una regadera de jardín y agua de acuerdo a la cantidad de semilla a aplicar; se tiene establecida una cantidad de 4 gramos de semilla para 180 bandejas (Protabaco, 2010); se llena la regadera con alrededor de 10 galones de agua y junto con estos se vierte la semilla, se disuelve por unos segundos y se procede a regar las bandejas de un lado a otro repitiendo las esquinas hasta terminar la mezcla de agua y semilla contenida en el recipiente; esto se hace para cada pasera que normalmente tiene de 180 a 200 bandejas, cantidad necesaria para sembrar una hectárea de tabaco.

Este método tradicional es el más usado ya que es rápido y efectivo pero genera altos costos en raleo, labor que consume alrededor de 5 jornales por Ha y nace a partir de la siembra en bandejas, el raleo consiste en retirar las plántulas demás que han germinado en los alveolos de tal manera que se deje una sola por celda, las plántulas retiradas se siembran en bandejas nuevas o son resembradas en alveolos en los que se perdió la semilla o no germino; este sobrecosto se genera debido a que la regadera no dosifica la cantidad de semilla que cae por alveolo originando un exceso de plántulas por bandeja; el raleo se realiza 20 a 25 días después de aplicada de semilla con una pinza, depilador o un palillo de dulce (Protabaco, 2009).

Para disminuir esta labor los agricultores han optado por sembrar de diferentes formas, una de estas es la siembra con jeringa de $5 \mathrm{ml}$ llenándola con varios gramos de semilla y golpeándola suavemente sobre cada alveolo con el fin de que caigan menos semillas en este; en cuanto a dosificación resulta un poco más efectivo que la siembra por regadera pero se convierte en un trabajo dispendioso cuando se realizan semilleros de más de $1 \mathrm{Ha}$, habituales en la zona debido a que se siembra tabaco rubio de clase Virginia, extensivo en comparación con el Burley o el tabaco negro para los cuales este método es muy beneficioso por ser siembras familiares en áreas reducidas. (Ministerio de agricultura y desarrollo rural, 2005)

Según Protabaco (2005), Otro método de siembra que se ha usado en la zona es el voleo, labor que se realiza al revolver 4 gramos de semilla con 500 gramos de arena o cal apagada, mezcla que se esparce por toda la pasera a una cantidad de 170 bandejas aproximadamente; para esta labor la semilla debe tener un mínimo de $80 \%$ de germinación. Es recomendable que el proceso completo de siembra se realice en forma continua y en lo posible en un solo lugar. Para el manejo de grandes cantidades de bandejas, existen en el mercado varios tipos de máquinas que permiten realizar estos pasos de una forma más o menos automatizada (Inta, 2003)

Barañao (1955) destaca la relevancia de la dosificación, debido a que el conjunto debe descargar una determinada cantidad de semilla, en forma constante y con independencia de su nivel dentro de la tolva. Los factores que influencian la precisión de la dosificación son: el tipo de dosificador (Tourn, et al., 1994) y las tasas de aplicación, peso y tamaño de la semilla (Atkins, 2004).

\section{Metodología}

\subsection{Localización}

La investigación se realizó en gran parte de la zona tabacalera del departamento del Huila, principalmente en los municipios de Rivera y Campoalegre por consiguiente la recolección de información y diseño de los implementos se hizo de acuerdo a estas condiciones de suelo y cultivo, se visitaron 30 lotes en esta zona sumando un área cercana a las 
100 hectáreas, en los cuales mediante análisis de los costos de producción y encuestas realizadas a los agricultores se destacó la labor de siembra de semilla desnuda en bandejas como uno de los procesos más importantes e ineficientes dentro del ciclo productivo del cultivo de tabaco.

Para la evaluación y comparación de los implementos de siembra de semilla se contó con 40 bandejas de 128 alvéolos, 20 para cada implemento sumando un área total de $6.6 \mathrm{~m} 2$. El predio donde se ubicó el ensayo se llama La Bastilla de la vereda Río Neiva, municipio de Campoalegre, con una extensión de $1 \mathrm{Ha}$, este lote presenta una pluviosidad de $1254 \mathrm{~mm} / \mathrm{año}$, en la zona la temperatura promedio es de $27^{\circ} \mathrm{C}$ con un clima cálido, la topografía es relativamente plana con leve pendiente y está ubicada a una altura de 525 m.s.n.m. (Datos estación meteorológica SENA. 2008).

\subsection{Caracterización de la semilla de tabaco}

Debido al diámetro mínimo de la semilla de tabaco y a la poca bibliografía encontrada en libros e internet sobre esta, se determinaron los parámetros necesarios para la evaluación y calibración del implemento. En la tabla 1 se resumen los datos generales de la semilla de tabaco utilizada en los ensayos.

Tabla 1. Datos generales de la semilla.

\begin{tabular}{|c|c|}
\hline Clase & Virginia estufado \\
\hline Variedad & $\mathrm{K}-399$ \\
\hline Cosecha & 2005 \\
\hline Procedencia & La floresta - san mateo \\
\hline Germinación & $75 \%$ \\
\hline
\end{tabular}

Inicialmente se tamizo la totalidad de la semilla para extraerle impurezas y grumos, luego se realizó el tamizado con un peso inicial de 5 gramos para un juego de tamices de granulometría de suelos, los resultados se resumen en la tabla 2.

Tabla 2. Determinación de diámetro promedio de la semilla.

\begin{tabular}{|c|c|c|c|}
\hline \# De tamiz & Abertura $(\mathbf{m m})$ & Semilla retenida $(\mathbf{g})$ & Porcentaje de retención \\
\hline 0 & 2 & 0 & $0 \%$ \\
\hline 20 & 0.85 & 0 & $0 \%$ \\
\hline 30 & 0.6 & 5 & $100 \%$ \\
\hline 50 & 0.3 & 0 & $0 \%$ \\
\hline 100 & 0.15 & 0 & $0 \%$ \\
\hline 200 & 0.075 & 0 & $0 \%$ \\
\hline
\end{tabular}

*Diámetro promedio de la semilla: $0,6 \mathrm{~mm}=600$ micras

Adicionalmente, se calculó el ángulo de deslizamiento de la semilla colocando 50 gr en una superficie plana y lisa e inclinándola suavemente hasta que esta comience a deslizarse, se calcula el ángulo formado con la horizontal y la altura de deslizamiento (Villamizar, 1993), este permite establecer la inclinación o talud de las paredes de la tolva, dato necesario para garantizar la evacuación de la totalidad de la semilla.

Tan $\alpha=$ altura / base

Tan $\alpha=5,5 / 7,5 \quad \alpha=36,25$ Grados (Angulo de deslizamiento).

Otra característica fundamental de la semilla es el ángulo reposo, pendiente máxima de una porción o arrume después de estabilizarse por sí misma sobre una superficie nivelada (Villamizar, 1993), del cono formado por la semilla se relaciona la base y la altura de la siguiente manera:

$\operatorname{Tan} \beta=$ altura $/$ base

$\operatorname{Tan} \beta=3 / 4,25 \quad \beta=35,22$ Grados (Angulo de reposo). 


\subsection{Diseño de la sembradora manual de plántulas de tabaco}

Las bandejas plásticas utilizadas para semilleros de tabaco tienen 8 alveolos a lo ancho por 16 alveolos a lo largo para un total de 128 , presentan además una pestaña de $0.5 \mathrm{~cm}$ por todo su contorno, cada alveolo tiene $2 \mathrm{~cm}$ de ancho por $4 \mathrm{~cm}$ de alto.

El diseño se realizó para abarcar lo ancho de la bandeja por lo tanto cuenta con 8 boquillas y 8 golpeadores de boquilla espaciados de acuerdo a la ubicación de los alveolos de tal manera que cada boquilla coincida con el centro de cada alveolo con el fin de depositar las semillas sin ningún tipo de obstáculo (Figura 1); el mango de tiro presenta un ángulo de 30 grados respecto a la horizontal como se ilustra en la figura 2, abertura ideal para minimizar el momento negativo que se genera al deslizar el implemento.

La sembradora manual de semilla desnuda trabaja por efecto del impacto de los golpeadores y la gravedad, por lo cual el diámetro de salida de las boquillas es de $1.0 \mathrm{~mm}, 0.4 \mathrm{~mm}$ por encima del promedio del diámetro de la semilla, holgura suficiente para la salida de estas hacia la bandeja.

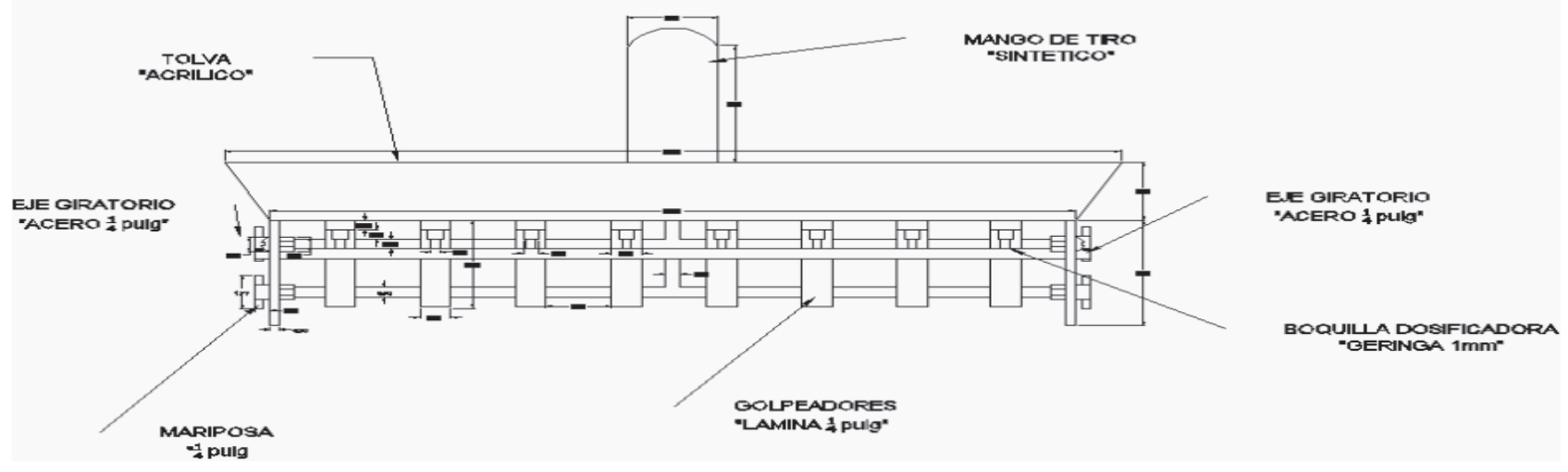

Figura 1. Vista frontal de sembradora manual de semilla desnuda.

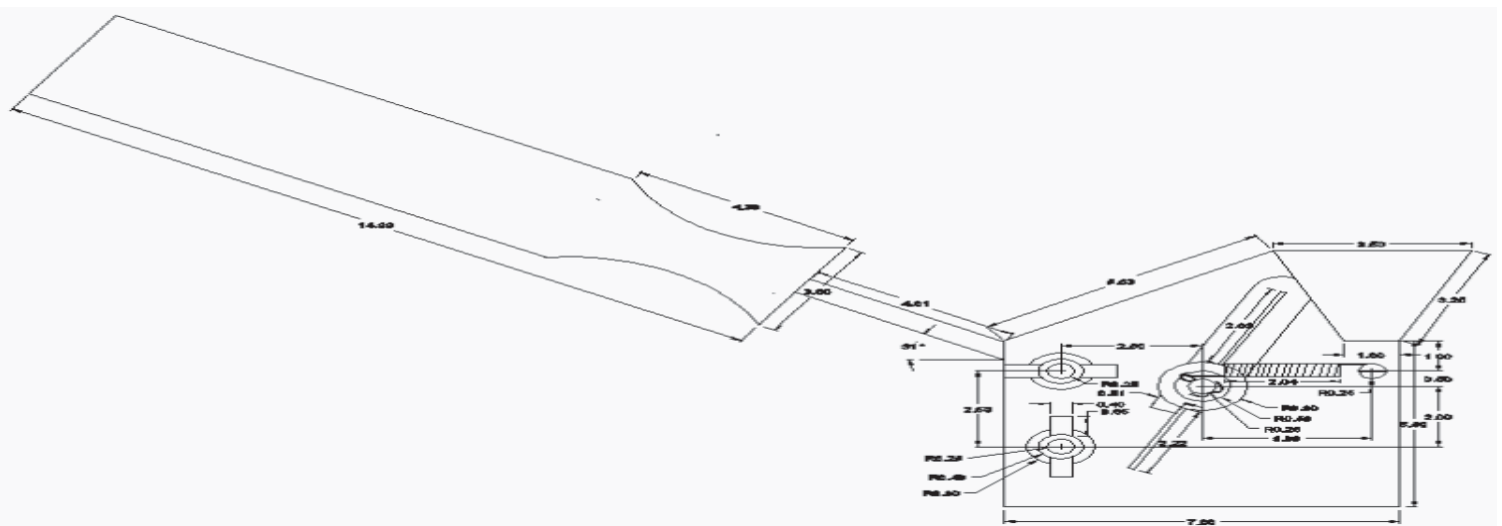

Figura 2. Vista Lateral de sembradora manual de semilla desnuda. 


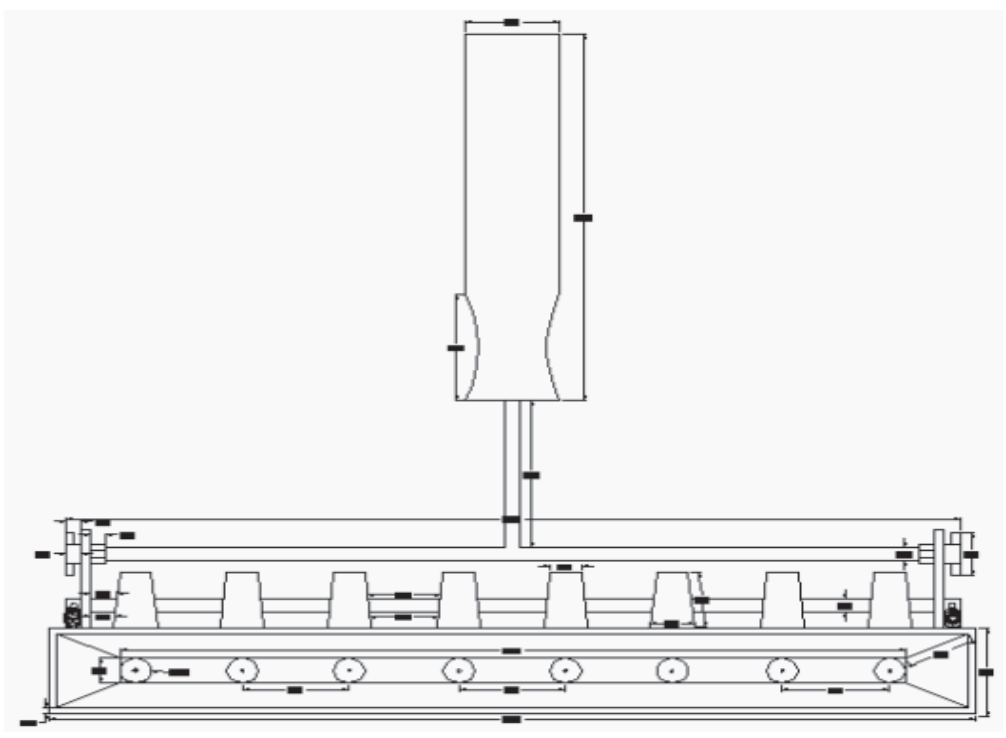

Figura 3. Vista superior de sembradora manual de semilla desnuda.

\subsection{Construcción de la sembradora manual de semilla desnuda}

Este implemento se construyó en acrílico de $3 \mathrm{~mm}$ de espesor para el caso de la tolva con su respectiva tapa y paredes laterales, los golpeadores y el bastidor se fabricaron en hierro, las boquillas y el mango se encontraron en el mercado regional en pasta.

Para la calibración de las boquillas se realizaron ensayos anteriores a la evaluación con jeringas de diámetros de salida de $0,6 \mathrm{~mm} 1,5 \mathrm{~mm}$; se colocaban en la sembradora y se hacia la labor de siembra sobre una bandeja vacía observando la cantidad de semillas depositadas mediante un papel que ilustra la disposición de los alveolos en la bandeja.

La caída de la semilla se realiza mediante la vibración que generan los golpeadores al chocar contra la tolva, estos golpes se producen por la fuerza contraria al movimiento de siembra proporcionada por los resortes anclados en las paredes laterales de la sembradora; por lo tanto, al calibrar las boquillas indirectamente se calibraron los resortes.

\subsection{Evaluación de la sembradora manual de semilla desnuda}

Los implementos evaluados están asociados al método de distribución de la semilla, regada para el implemento I y puntual por alveolo para el implemento II.

I:Tradicional con regadera.

II: Sembradora manual.

\subsubsection{Tamaño del ensayo}

40 bandejas de 128 alvéolos.

- Numero de parcelas: 2

- Parcela: 20 bandejas

- Número de plantas por implemento: 2560

- Número de bandejas por implemento: 20

- Número de plantas total del ensayo: 5120

- Número de implementos: 2 
Entre los materiales relevantes en la implementación del semillero se tienen bandejas plásticas marca Poliexpandibles utilizadas en cosechas anteriores para este lote, sustrato a base de turba negra marca Klasman y paseras en guadua realizadas para semilleros de cosechas pasadas en las que se distribuyeron las bandejas como se ilustra en la figura 4.

\section{IMPLMENTO I \\ IMPLEMENTO II}

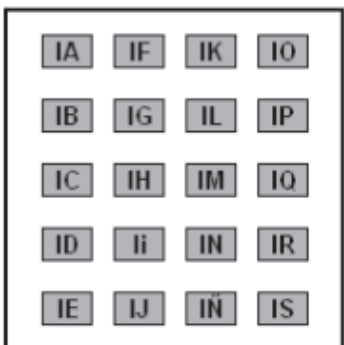

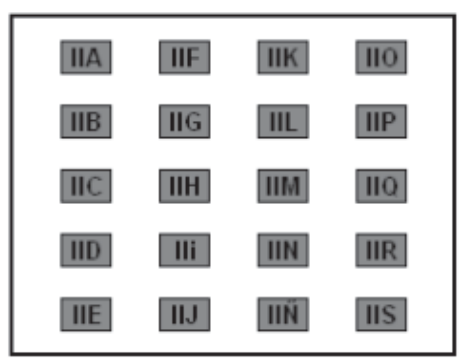

Figura 4. Esquema de evaluación para los implementos de siembra.

Al reutilizar bandejas para la evaluación se debió realizar un lavado exhaustivo con abundante agua con el fin de retirar residuos de turba y/o raíces de semilleros anteriores que puedan llevar cualquier tipo de plaga o enfermedad que altere los resultados a la hora de germinar la semilla depositada con este implemento.

\subsubsection{Variables a Evaluar.}

Para la evaluación preliminar de la sembradora manual se tuvo en cuenta las siguientes variables de respuesta:

- Numero de semillas depositadas por alvéolo. (SEM*ALV)

- Tiempo de duración de la labor. (t LABOR (seg))

- Alveolos sin semilla. (ASS)

- Porcentaje de germinación. (\% GER)

- Plántulas a ralear

\section{Resultados}

Producto de la evaluación, en la tabla 3 se resumen los datos registrados al comparar el desempeño de la siembra tradicional por regadera frente a la sembradora manual de semilla desnuda.

Tabla 3. Variables de respuesta implementos de siembra de semilla

\begin{tabular}{|c|c|c|c|c|c|}
\hline Implemento & T Labor(Seg) & Sem*Alv. & Ass & \% Ger. & Plántulas a Ralear \\
\hline Implemento I & 178,2 & 5,8 & 174 & 88,4 & 12288 \\
\hline Implemento II & 88,4 & 39,92 & 383 & 83,3 & 2549,76 \\
\hline
\end{tabular}

Se observa una disminución del $36 \%$ en la cantidad de semillas depositadas por alveolo, cifra muy significativa ya que este parámetro es el que genera la cantidad de plántulas a ralear que para la sembradora manual fue de 2549 comparada con las 12288 de la siembra por regadera; se resalta también una disminución del $50.4 \%$ en el tiempo total de la labor (Figura 5) viéndose reflejado en la disminución de jornales y por ende en los costos del proceso de siembra de semilla en bandejas. 


\section{SEMBRADORA MANUAL Vs TRADICIONAL CON REGADERA.}

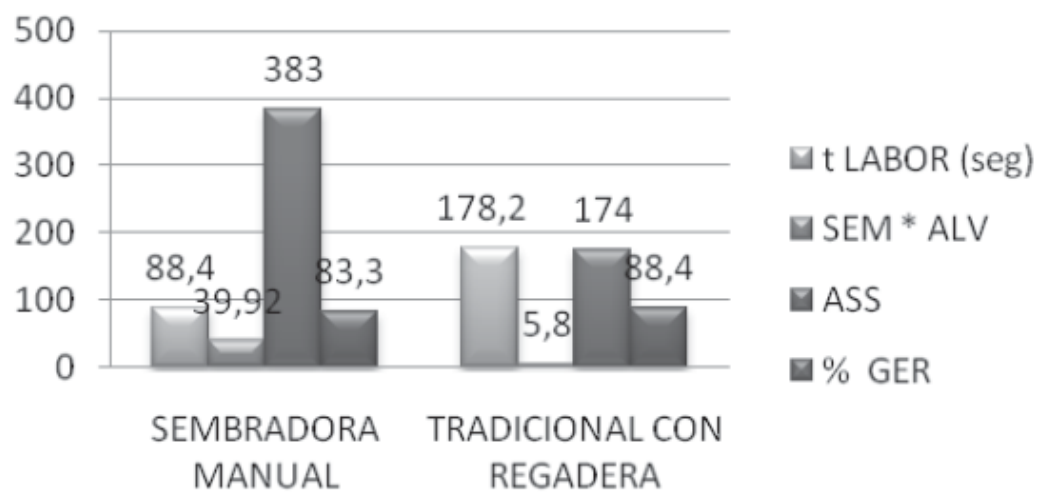

Figura 5. Comportamiento de variables de respuesta para los implementos de siembra de semilla

En el raleo se fundamenta la adquisición del implemento para los agricultores, no se concibe un adelanto en el método de siembra de semilla sin que este incida de manera positiva en la disminución de esta labor; en la figura 6 se observa una reducción del 79,3 \% en la cantidad de plántulas a ralear para 20 bandejas sembradas con la sembradora manual, cifra bastante diciente frente al método tradicional por regadera.

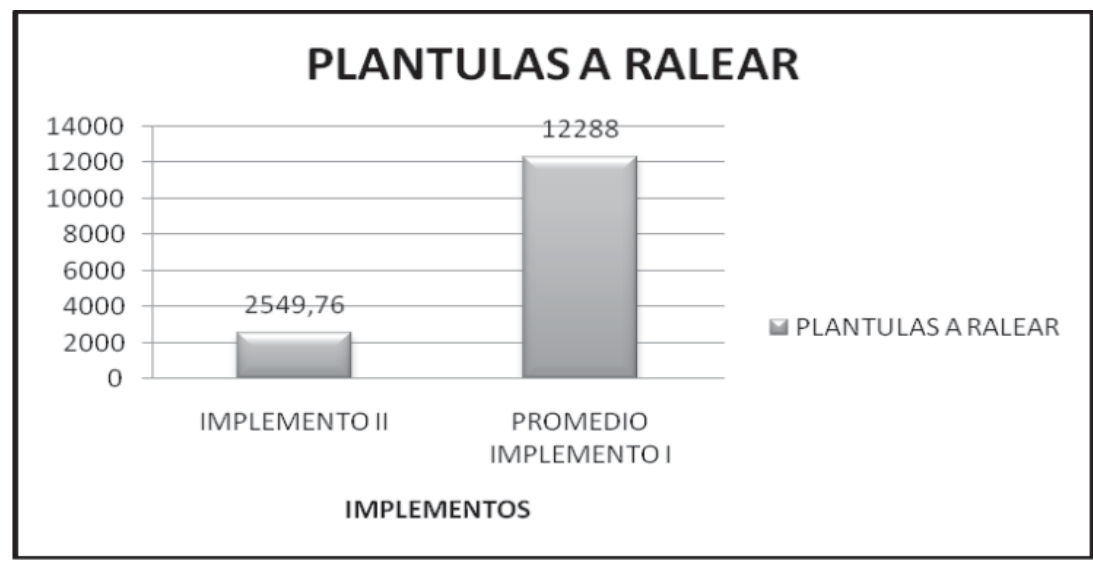

Figura 6. Plántulas a ralear por implemento

\section{Conclusiones.}

En la caracterización de la semilla de tabaco para la variedad K - 399 se encontró un diámetro promedio de 600 micras o 0,6 mm, un ángulo de deslizamiento es de 36,25 grados y un ángulo de reposo es de 35,22 grados.

El porcentaje de germinación para la siembra por regadera muestra un nivel de 5,1\% por encima de la sembradora manual, esto se debe a que la semilla está mezclada con agua y no sufre mayores daños mecánicos en la regadera a diferencia de la sembradora manual ya que esta presenta boquillas de diámetro reducido, una tolva desnuda y movimientos repetitivos que generan ciertos daños. 
La gran falencia del método de siembra por regadera radica en el desperdicio de semilla al ser aplicada, puesto que no presenta ningún tipo de dosificación y además la riega no se hace por bandeja sino que se realiza por pasera de 180 o 200, este montaje deja espacios entre bandejas por donde se escurre la mezcla de agua con semilla generando pérdidas de tiempo y dinero.

La sembradora manual otorga una disminución del $36 \%$ en la cantidad de semillas depositadas por alveolo, cifra muy significativa ya que este parámetro es el que genera la cantidad de plántulas a ralear que para esta fue de 2549 comparada con las 12288 de la siembra por regadera o convencional.

La sembradora manual presenta una disminución del $50.4 \%$ en el tiempo total de la labor y una reducción del 79,3\% en la cantidad de plántulas a ralear para 20 bandejas sembradas con la sembradora manual, cifra bastante diciente frente al método tradicional por regadera.

En cuanto al dinero gastado en la labor de siembra de semilla desnuda en bandejas, la sembradora manual construida mostro un ahorro del $44 \%$ comparado con la siembra tradicional con regadera.

\section{Referencias Bibliográficas}

1. Atkins, R. P. 2004. Accurate Metering of Seed and Fertilizer. Alberta Farm Machinery Center. Lethbridge, Alberta. En: www1.agric.gov.ab.ca/departament/deptdocs.nst/all/eng8023.

2. Barañao, T. V. 1955. Maquinaria Agrícola. Salvat Editores S.A. Barcelona. España. 608 pp.

3. Instituto Nacional de Tecnología Agropecuaria INTA. 2003. Producción de plantas de tabaco en bandejas flotantes / Proyecto P94 PROZONO: Alternativas al bromuro de metilo. Buenos Aires: Ediciones INTA, 139 p.

4. Ministerio de Agricultura y Desarrollo Rural. 2005. Observatorio Agrocadenas Colombia. La cadena del tabaco en Colombia. "Una mirada global de su estructura y dinámica 1991-2005”. Documento de Trabajo No. 55. Marzo, 2005.

5. Protabaco Ltda. 2005. Módulos de Siembra, Mantenimiento, Recolección y Curado del cultivo de tabaco. Curso de capacitación agricultores y aprendices. SENA. Santander. pp. 1-50

6. Protabaco Ltda.2009. Presentación Huila. Neiva - Huila, 12 p.

7. Protabaco Ltda.2010. Presentación semilleros y preparación de suelos. San Gil - Santander, $44 \mathrm{p}$.

8. Tourn, M. C., E. L. Soza y A. Mete. 1994. Cuantificación del tratamiento que otorgan a la semilla de soja dos dosificadores de sembradoras para cultivos en masa, en hileras. I Congreso Internacional de Ingeniería Agrícola. Chillán, Chile. 6 pp.

9. Villamizar, Fanny.1993. Manual de prácticas de procesos agrícolas. Universidad Nacional de Colombia (sede Bta.) Facultad de ingeniería. Bogotá - Colombia. 180 p. 